\title{
Zoogeographical relationships of the littoral ascidiofauna around the Antarctic Peninsula, in the Scotia Arc and in the Magellan region*
}

\author{
ALFONSO A. RAMOS-ESPLÁ, JUAN A. CÁRCEL and MERCEDES VARELA \\ Marine Biology Laboratory, Department of Marine Sciences and Applied Biology, University of Alicante, \\ E-03080 Alicante, Spain. E-mail: Alfonso.Ramos@ua.es
}

\begin{abstract}
SUMMARY: Three Spanish Antarctic research cruises (Ant-8611, Bentart-94 and Bentart-95) were carried out in the South Shetland Archipelago (Antarctic Peninsula) and Scotia Arc (South Orkney, South Sandwich and South Georgia archipelagos) on the continental shelf and upper slope (10-600 $\mathrm{m}$ depth). They have contributed to our knowledge about ascidian distribution and the zoogeographical relationships with the neighbouring areas and the other Subantarctic islands. The distribution of ascidian species suggests that the Scotia Arc is divided into two sectors, the South Orkney Archipelago, related to the Antarctic Province, and the South Georgia Archipelago (probably including the South Sandwich Archipelago), which is intermediate between the Antarctic Province and the Magellan region.
\end{abstract}

Keywords: ascidians, Antarctic, Scotia Arc, Magellan, biogeography.

RESUMEN: RELACIONES ZOOGEOGRÁFICAS DE LA ASCIDIOFAUNA LITORAL EN LA PENÍNSULA ANTÁRTICA, ARCO DE SCOTIA Y REGIÓN MAGALLÁNICA. - Tres campañas antárticas españolas (Ant-86, Bentart-94 y Bentart-95) en el Archipiélago de las Shetland del Sur (Península Antártica) y Arco de Scotia (Archipiélagos de las Órcadas del Sur, Sandwich del Sur y Georgia del Sur), sobre fondos de la plataforma continental y parte superior del talud (profundidades entre 10 y $600 \mathrm{~m}$ ), han contribuido a ampliar el conocimiento de la distribución de las ascidias en estas zonas, y sus relaciones biogeográficas con las áreas vecinas y otras islas subantárticas. Desde el punto de vista de la ascidiofauna, el Arco de Scotia se divide en dos sectores, por un lado las Órcadas del Sur más relacionadas con la Provincia Antártica; y por otro el Archipiélago de las Georgias del Sur (y probablemente las islas Sandwich del Sur) que ocupa una posición intermedia entre la Provincia Antártica y la región magallánica.

Palabras clave: ascidias, Antártida, Arco de Scotia, Magallanes, biogeografía.

\section{INTRODUCTION}

The Antarctic Province represents one of the most clearly defined biogeographic divisions in the world. Its northern limit is defined by the Antarctic Convergence (Ekman, 1967, Hedgpeth, 1969; Briggs, 1974; Knox, 1994; Crame, 1999). However, there are several hypotheses about the subdivisions

*Received April 26, 2004. Accepted March 10, 2005. of the Antarctic region, and their relationships with the Scotia Arc and the Magellan region. Ekman (1967) and Briggs (1974) consider two main subdivisions (subregions of Ekman, and provinces sensu Briggs). The latter proposed: i) a high Antarctic subregion or South Polar Province containing the continental coastal area and adjoining islands (including the South Shetland, South Orkney and South Sandwich archipelagos); and ii) a low Antarctic subregion or South Georgia Province containing the 
South Georgia Archipelago and the Shag Rock Bank. Dell (1972) opposed this hypothesis on the relationships of the Antarctic benthic fauna, emphasising that there is little evidence to warrant biogeographic subdivisions. Recently, Knox (1994), following Hedgpeth's scheme (1969), considered the Subantarctic region to be divided into the Magellan and Kerguelen Provinces or subregions; the Antarctic region in the western Antarctic, with the Antarctic Peninsula and the Scotia Arc (with the South Georgia district); and the Continental Antarctic. Longhurst (1998), basing his conclusions on plankton ecology, proposed that the Scotia Arc and the South Georgia Archipelago be included in the Austral Polar Province (Antarctic continent and adjoining islands). Arntz (1999) stressed the necessity of more effort in taxonomy to improve knowledge on floral and faunal relationships between the Antarctic and Magellan regions.

From a biogeographical point of view, the ascidians represent an excellent taxon for studying possible affinities between zones (Kott, 1969; Millar, 1971; Monniot and Monniot, 1983; RamosEsplá, 1991), and they have a high percentage of endemisms in the Antarctic region (about 34-38\% in the Antarctic and Subantarctic regions in Monniot and Monniot, 1983). In terms of numbers and biomass, ascidians represent one of the main sessile benthic groups on the Antarctic continental shelf (Kott, 1969; Monniot and Monniot, 1983; Arnaud et al., 1998), and they play an important role in the structure of filter-feeding communities (Gallardo, 1987; Jazdzewski et al., 1986; Dayton, 1990; Galéron et al., 1992; Arntz et al., 1994, 1997; Sahade et al., 1998; Kowalke, 1999; Gili et al., 2001).

The Antarctic ascidiofauna has been well studied (see references in Van Name, 1945; Millar, 1960; Kott, 1969; Monniot and Monniot, 1983), and recent studies have focused on the South Shetland Archipelago and Weddell Sea (Rauschert, 1991; Monniot and Monniot, 1994; Luján and Ramos-Esplá, 1996; Tatián et al., 1998; Varela and Ramos-Esplá, 2003). Also, the Scotia Arc and the Magellan region have been the subject of recent ascidian studies (Sanamyan and Schories, 2003). (Tatián and Sahade in Arntz and Brey, 2003). This study explores the biogeographical relationships of the Antarctic Peninsula and Scotia Arc with the Antarctic continent, Magellan region and the Subantarctic islands from the point of view of littoral ascidiofauna.

\section{MATERIAL AND METHODS}

Ascidians were collected (Table 1) on three Spanish Antarctic cruises: Ant-8611 (Scotia Arc and Elephant Island) with the fisheries vessels 'Pescapuerta IV' and 'Nuevo Alcocero'; and Bentart-94 (Livingston and Deception Islands) and Bentart-95 (Livingston, Deception and Trinity Island, and Bransfield Strait) with the RV 'Hespérides'. The samples were taken from the continental shelf and from upper slope locations, between 10 and $600 \mathrm{~m}$ depth, by SCUBA diving and remote sampling gears (Agassiz trawl, rocky and anchor dredges and Van Veen grab). Macrobenthos $(\varnothing \geq 1 \mathrm{~mm})$ was sorted by sieve and the ascidians were separated, anaesthetised in menthol, and fixed/preserved in neutral formalin (10\% in sea water with borax).

With regard to the ascidian distribution, we considered the following zones: i) the Antarctic region separating the Antarctic Peninsula (Graham and Palmer Lands, and adjoining islands, including the South Shetland Archipelago) from the Antarctic Continent; ii) the Scotia Arc, including the South Orkney, South Sandwich and South Georgia archipelagos; iii) the Magellan region, south of a line from Chiloé Island (at $42^{\circ} \mathrm{S}$ ) to the mouth of the Rio de la Plata (at $35^{\circ} \mathrm{S}$ ); and iv) the Subantarctic islands (Bouvet, Prince Edwards, Marion, Crozet, Kerguelen, Heard, MacDonald, Macquarie). For the purpose of distributional analysis we considered the shore or littoral ascidiofauna extending from the surface down to $500 \mathrm{~m}$ depth. This arbitrary depth was selected because the Antarctic ascidian fauna is rather uniform over this range (Briggs, 1974) and some deep Antarctic ascidian species such as Cibacapsa gulosa reach 500-600 m depth (Monniot and Monniot, 1983, 1994).

The presence/absence data were aggregated by the Bray-Curtis similarity index, and the analysis of the relationships between species and zones was carried out by hierarchical agglomerate (group average) clustering for the species and multidimensional scaling analysis (MDS) for the zones indicated above: the Antarctic Continent (AC), the Antarctic Peninsula (AP), the South Orkney (SO), South Sandwich (SS) and South Georgia (SG) archipelagos, the Magellan region (MR) and the Subantarctic islands (SAI), using the PRIMER software package (Clarke and Warwick, 1994). With regard to the ascidian distribution, we followed mainly the work of Monniot and Monniot (1983: Table IV) and completed it with recent data. 


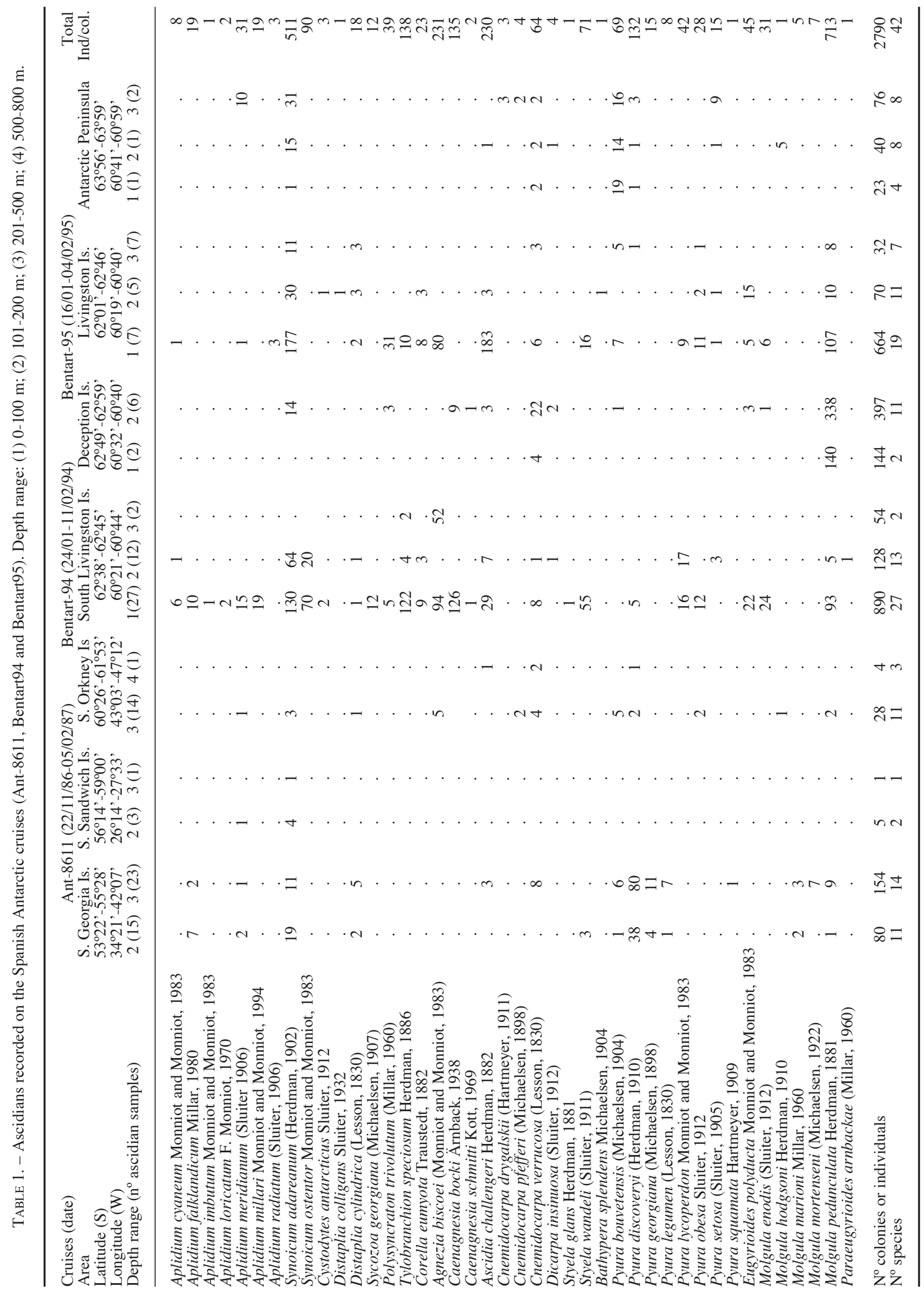




\section{RESULTS AND DISCUSSION}

\section{Species groups}

On the three Spanish Antarctic cruises Ant-8611, Bentart-94 and Bentart-95, forty-two species (Table 1) with about 2800 specimens were identified. Some species have enlarged their distribution zones: i) Synoicum adareanum, Styela wandeli, Pyura bouvetensis, P. legumen, P. squamata, Molgula marioni and $M$. mortenseni in South Georgia; ii) Aplidium millari and Synoicum ostentor in the South Shetlands (Antarctic Peninsula); iii) Synoicum adareanum and Aplidium meridianum in the South Sandwich Islands; and iv) Agnesia biscoei, Cnemidocarpa pfefferi and Pyura obesa in the South Orkney Islands.

The species cluster analysis (Fig. 1) from the sampled ascidians, with the records (Table 2) of different authors (Millar, 1960; Kott, 1969, 1971; Monniot and Monniot, 1983; 1994; Sanamyan and Schories, 2003), discriminates two main groups of species (similarity < 40\%): I) the South GeorgiaMagellan group (4 spp, $9.5 \%$ of the total), Pyura legumen, Molgula marioni and M. mortenseni, and Pyura georgiana (an endemic species to South Georgia); and II) species with Antarctic distribution (38 spp., $90.5 \%$ of the total), some of them present in the Magellan region, the Scotia Arc and/or the Subantarctic islands.

In this second group three subgroups can be distinguished:

IIa) Species only found in the Antarctic Peninsula: Aplidium radiatum, Cystodytes antarcticus and Pyura lycoperdon.

IIb) Species found off the Antarctic Continent, the Antarctic Peninsula, the South Orkney and/or the South Sandwich archipelagos: Aplidium cyaneum, A. loricatum, Aplidium millari, Synoicum ostentor, Agnesia biscoei, Caenagnesia schmitti, Pyura obesa, P. setosa, and Molgula enodis and Paraeugyroides arnbackae, with Styela glans and Bathypera splendens (euribathyc species also reported in the Magellan region).

IIc) Species present in the Antarctic Region, the Scotia Arc, including South Georgia Archipelago (Caenagnesia bocki, Cnemidocarpa pfefferi, Dicarpa insinuosa, Syela wandeli, Pyura discoveryi, Eugyroides polyducta and Molgula hodgsoni), and some of them with a wide distribution in the Magellan Region and/or in the rest of the Subantarctic islands (Aplidium falklandicum, A. imbutum, A. meridianum, Synoicum adareanum, Distaplia colligans, D. cylindrica, Sycozoa georgiana, Polysyncraton trivolutum, Tylobranchion speciosum, Corel-

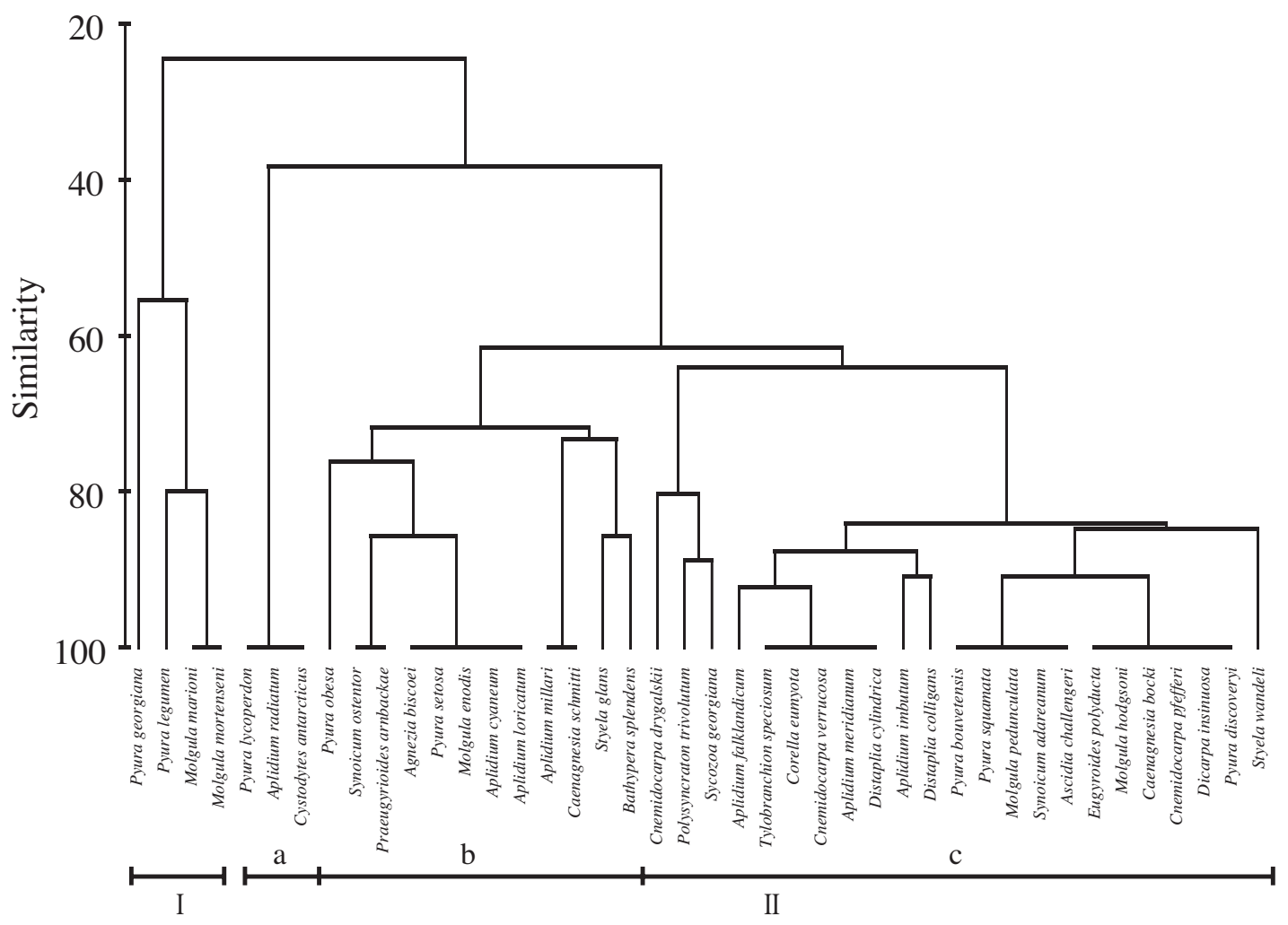

FIG. 1. - Cluster dendrogram of the sampled ascidian species with regard to the biogeographical affinities. 
TABLE 2. - Distribution of the Antarctic and Subantarctic ascidiofauna: (AC) Antarctic continent; (AP) Antarctic Peninsula; (MR) Magellan region; (SAI) Subantarctic islands (Gough, Bouvet, Prince Edward, Marion, Crozet, Kerguelen, Heard, McDonald, Macquarie); (SG) South Georgia Is.; (SO) South Orkney Is.; (SS) South Sandwich Is.. (Dr) depth range: (1) 0-200 m; (2) 201-500 m; (3) 501-1000 m); (4) > $1000 \mathrm{~m}$. (AZ) other zones: (1) Chile $\left(<42^{\circ} \mathrm{S}\right)$, Peru $\left(>17^{\circ} \mathrm{S}\right)$; (2) New Zealand sector $\left(<50^{\circ} \mathrm{S}\right)$; (3) Tasmania, Southern Australia; (4) South Africa, Namibia; (5) Cosmopolitan.

\begin{tabular}{llllllllllllllllllll}
\hline MR & SG & SS & SO & AP & AC & SAI & Dr & AZ &
\end{tabular}

Fam. Polyclinidae Verrill, 1871

Aplidiopsis discoveryi Millar, 1960

Aplidiopsis pyriformis Herdman, 1886

Aplidium acropodium Monniot and Gail, 1978

Aplidium annulatum Sluiter, 1906

Aplidium aurorae (Harant and Vernières, 1938)

Aplidium balleniae Monniot and Monniot, 1983

Aplidium bilinguae Monniot and Monniot, 1983

Aplidium circumvolutum (Sluiter, 1900)

Aplidium cyaneum Monniot and Monniot, 1983

Aplidium didemniformis Monniot and Gail, 1978

Aplidium falklandicum Millar, 1960

Aplidium fuegiense Cunninghan, 1871

Aplidium globosum (Herdman, 1886)

Aplidium gracile Monniot and Monniot, 1983

Aplidium hians Monniot and Gail, 1978

Aplidium imbutum Monniot and Monniot, 1983

Aplidium irregulare (Herdman, 1886)

Aplidium laevigatum Herdman, 1886

Aplidium leviventer Monniot and Gail, 1978

Aplidium longum F.Monniot, 1970

Aplidium loricatum (Harant and Vernières, 1938)

Aplidium magellanicum Sanamyan and Schories, 2003

Aplidium meridianum, (Sluiter, 1906)

Aplidium millari Monniot and Monniot, 1994

Aplidium miripartum Monniot and Monniot, 1983

Aplidium nottii (Brewin, 1951)

Aplidium novaezealandiae Brewin, 1952

Aplidium ordinatum (Sluiter, 1906)

Aplidium ovum F. Monniot and Gaill, 1978

Aplidium paessleri (Michaelsen, 1907)

Aplidium pellucidum Kott, 1971

Aplidium pererratum (Sluiter, 1912)

Aplidium peresi $\mathrm{F}$. Monniot, 1970

Aplidium quadriversum Millar, 1982

Aplidium radiatum (Sluiter, 1906)

Aplidium recumbens (Herdman, 1886)

Aplidium retiforme (Herdman, 1886)

Aplidium siderum Monniot and Monniot, 1983

Aplidium stanleyi Millar, 1960

Aplidium stewartense (Michaelsen, 1924)

Aplidium triplex (Sluiter, 1906)

Aplidium undulatum F. Monniot and Gaill, 1978

Aplidium vanhoffeni Hartmeyer, 1911

Aplidium variabile (Herdman, 1886)

Aplidium vastum (Sluiter, 1912)

Aplidium vexillum Monniot and Gail, 1974

Placentella translucida Kott, 1969

Polyclinum sluiteri Brewin, 1956

Ritterella mirifica Monniot and Monniot, 1983

Synoicum adareanum (Herdman, 1902)

Synoicum georgianum Sluiter, 1932

Synoicum giardi (Herdman, 1886)

Synoicum kerguelenense Hartmeyer, 1911

Synoicum ostentor Monniot and Monniot, 1983

Synoicum polygyna Monniot and Monniot, 1980

Synoicum ramulosum Kott, 1969

Synoicum salivum Monniot and Gail, 1978

Fam. Didemnidae Giard, 1872

Didemnum biglans (Sluiter, 1906)

Didemnum studeri Hartmeyer, 1911

Didemnum subflavum (Herdman, 1886)

Didemnum tenue (Herdman, 1886)

Diplosoma antarcticum Kott, 1969

Diplosoma longinquum (Sluiter, 1912)

Leptoclinides capensis (Michaelsen, 1934)

Leptoclinides kerguelenensis Kott, 1954

Polysyncraton trivolutum (Millar, 1960)

Trididemnum auriculatum (Michaelsen, 1934)

Trididemnum propinquum (Herdman, 1886)

\begin{tabular}{|c|c|c|c|c|c|c|c|c|}
\hline+ & . & • & - & . & . & • & 1 & 2 \\
\hline • & . & • & . & . & . & + & 1 & . \\
\hline . & . & $\cdot$ & . & • & . & + & 1 & . \\
\hline . & • & . & . & + & . & . & 1 & . \\
\hline . & • & . & . & - & + & • & $1-2$ & . \\
\hline$\cdot$ & • & . & • & . & + & . & 1 & 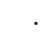 \\
\hline . & + & • & . & . & + & . & $1-2$ & \\
\hline+ & + & . & . & + & . & + & $1-2$ & 1 \\
\hline . & • & . & + & + & + & . & $1-4$ & . \\
\hline . & • & . & . & $\cdot$ & . & + & 1 & . \\
\hline+ & + & . & + & + & . & + & $1-2$ & \\
\hline+ & + & · & + & + & + & + & $1-3$ & 1 \\
\hline$\cdot$ & . & + & + & . & . & + & $1-3$ & . \\
\hline+ & . & • & . & . & . & . & $1-3$ & . \\
\hline . & . & • & • & . & . & + & 1 & . \\
\hline+ & + & . & + & + & + & . & $1-3$ & . \\
\hline+ & . & • & . & . & . & . & $1-3$ & . \\
\hline+ & . & . & . & . & . & + & 1 & . \\
\hline . & . & • & • & . & . & + & 1 & . \\
\hline+ & . & . & . & . & . & + & 1 & . \\
\hline . & . & . & + & + & + & . & $1-3$ & . \\
\hline+ & . & . & . & . & . & . & 1 & \\
\hline+ & + & + & + & + & + & + & $1-4$ & . \\
\hline . & . & . & . & + & + & . & $1-2$ & . \\
\hline . & . & . & . & + & . & . & $1-2$ & \\
\hline . & • & • & . & . & . & + & 1 & 2 \\
\hline . & . & • & . & . & • & + & 1 & 2 \\
\hline . & • & . & . & + & + & . & $1-2$ & \\
\hline+ & . & . & . & . & . & + & $1-2$ & 3 \\
\hline+ & + & . & . & . & • & . & 1 & . \\
\hline+ & . & . & . & . & . & . & 1 & ${ }^{\circ}$ \\
\hline . & . & + & + & + & + & . & $1-2$ & . \\
\hline • & . & • & . & . & . & + & 1 & . \\
\hline . & • & • & • & . & . & + & 1 & . \\
\hline · & • & · & . & + & . & . & 1 & . \\
\hline+ & . & + & + & + & . & . & $1-2$ & . \\
\hline . & . & . & . & . & . & + & 1 & . \\
\hline : & : & • & • & + & - & - & 1 & . \\
\hline+ & + & . & . & + & . & . & $1-2$ & \\
\hline+ & + & . & . & . & . & + & 1 & 2 \\
\hline+ & . & . & . & + & . & . & $1-2$ & . \\
\hline+ & . & . & . & . & . & + & 1 & . \\
\hline . & . & • & . & . & + & . & 2 & \\
\hline+ & + & . & . & . & . & + & $1-2$ & 2 \\
\hline . & . & . & . & + & . & . & 1 & . \\
\hline . & . & • & • & . & • & + & 1 & . \\
\hline · & • & • & . & + & • & - & 2 & \\
\hline+ & . & . & . & . & . & . & 2,3 & 2 \\
\hline . & . & . & . & + & + & . & 2 & . \\
\hline . & + & + & + & + & + & + & $1-3$ & . \\
\hline+ & + & + & + & + & + & + & $1-2$ & . \\
\hline+ & + & • & • & . & . & + & 1 & . \\
\hline • & . & · & . & • & - & + & 1 & . \\
\hline • & . & + & . & + & + & . & $1-2$ & . \\
\hline • & • & • & - & • & + & - & 2 & . \\
\hline . & . & . & . & . & + & . & 1 & . \\
\hline$\cdot$ & • & $\cdot$ & $\cdot$ & $\cdot$ & - & + & 1 & . \\
\hline . & + & + & + & + & + & . & $1-4$ & 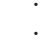 \\
\hline+ & + & . & + & • & . & + & $1-4$ & 2,3 \\
\hline & 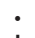 & · & • & . & . & + & 1 & . \\
\hline+ & + & . & . & . & . & . & $2-4$ & . \\
\hline$\cdot$ & • & • & - & + & + & - & 1 & $\cdot$ \\
\hline • & + & . & . & + & . & . & $1-2$ & \\
\hline . & • & • & • & . & - & + & 1 & 4 \\
\hline • & . & $\cdot$ & - & . & - & + & 1 & . \\
\hline+ & + & . & . & + & + & + & $1-3$ & . \\
\hline+ & . & ${ }^{\circ}$ & . & $\cdot$ & • & • & 1 & . \\
\hline+ & . & . & . & . & . & . & 2 & . \\
\hline
\end{tabular}


TABle 2 (CONT.). - Distribution of the Antarctic and Subantarctic ascidiofauna: (AC) Antarctic continent; (AP) Antarctic Peninsula; (MR) Magellan region; (SAI) Subantarctic islands (Gough, Bouvet, Prince Edward, Marion, Crozet, Kerguelen, Heard, McDonald, Macquarie); (SG) South Georgia Is.; (SO) South Orkney Is.; (SS) South Sandwich Is.. (Dr) depth range: (1) 0-200 m; (2) 201-500 m; (3) 501-1000 m); (4) $>1000$ m. (AZ) other zones: (1) Chile $\left(<42^{\circ} \mathrm{S}\right)$, Peru $\left(>17^{\circ} \mathrm{S}\right)$; (2) New Zealand sector $\left(<50^{\circ} \mathrm{S}\right)$; (3) Tasmania, Southern Australia; (4) South Africa, Namibia; (5) Cosmopolitan.

\begin{tabular}{|c|c|c|c|c|c|c|c|c|c|}
\hline & MR & SG & SS & SO & $\mathrm{AP}$ & $\mathrm{AC}$ & SAI & $\mathrm{Dr}$ & $\mathrm{AZ}$ \\
\hline \multicolumn{10}{|l|}{ Fam. Polycitoridae Michaelsen, 1904} \\
\hline Cystodites antarcticus Sluiter, 1912 & . & . & . & . & + & . & . & $1-2$ & . \\
\hline Distaplia colligans Sluiter, 1932 & + & + & . & + & + & . & . & $1-2$ & . \\
\hline Distaplia concreta (Herdman, 1886) & . & . & . & . & . & . & + & 1 & . \\
\hline Distaplia cylindrica (Lesson, 1830) & + & + & + & + & + & + & + & $1-3$ & . \\
\hline Eudistoma australe F. Monniot, 1978 & . & . & . & . & . & . & + & 1 & . \\
\hline Polycitor magalhaensis (Michaelsen, 1907) & + & . & + & + & . & . & . & 1 & \\
\hline Sycozoa anomala Millar, 1960 & . & . & . & . & + & . & . & 1 & 2 \\
\hline Sycozoa gaimardi (Herdman, 1886) & + & + & . & . & + & + & $\dot{.}$ & $1-2$ & . \\
\hline Sycozoa georgiana (Michaelsen, 1907) & . & + & . & . & + & + & + & $1-2$ & \\
\hline Sycozoa sigillinoides Lesson, 1830 & + & + & + & . & + & + & + & $1-3$ & 2 \\
\hline Tetrazona glareosa (Sluiter, 1906) & . & . & + & $\dot{0}$ & + & . & . & 1 & . \\
\hline \multicolumn{10}{|l|}{ Fam. Cionidae Lahille, 1890} \\
\hline Tylobranchion speciosum Herdman, 1886 & + & + & + & + & + & + & + & $1-2$ & . \\
\hline Ciona antarctica Hartmeyer, 1911 & . & . & . & . & + & + & . & $1-2$ & \\
\hline ?Ciona intestinalis (Linnaeus, 1767) & + & . & . & . & . & . & . & $1-2$ & 5 \\
\hline \multicolumn{10}{|l|}{ Fam. Corellidae Lahille 1890} \\
\hline Corella eumyota Traustedt, 1882 & + & + & . & + & + & + & + & $1-3$ & $1,2,3,4$ \\
\hline Corynascidia cubare Monniot and Monniot, 1994 & . & . & . & . & . & + & . & 2 & . \\
\hline Xenobranchion insigne Ärnbäck-Christie-Linde, 1950 & + & . & : & . & $\dot{0}$ & . & . & 1 & . \\
\hline \multicolumn{10}{|l|}{ Fam. Agneziidae Hunstsman, 1912} \\
\hline Adagnesia henriquei Monniot and Monniot, 1983 & + & . & . & . & . & . & . & 1 & . \\
\hline Adagnesia weddelli Monniot and Monniot, 1994 & . & : & : & . & . & + & . & $2-4$ & . \\
\hline Agnezia antarctica Kott, 1969 & . & . & . & . & . & . & + & 1 & . \\
\hline Agnezia arnaudi (Monniot and Monniot, 1974) & . & . & + & . & . & + & + & 1 & . \\
\hline Agnezia biscoei (Monniot and Monniot, 1983) & . & . & + & + & + & + & . & 1 & . \\
\hline Agnezia glaciata (Michaelsen, 1898) & + & $\dot{.}$ & . & . & . & . & . & 1 & . \\
\hline Agnezia tenue (Monniot and Monniot, 1983) & + & . & . & . & . & . & . & 1 & . \\
\hline Caenagnesia bocki Ärnback, 1938 & . & + & + & . & + & + & . & $1-3$ & . \\
\hline Caenagnesia schmitti Kott, 1969 & $\dot{.}$ & . & . & . & + & + & . & $1-4$ & . \\
\hline \multicolumn{10}{|l|}{ Fam. Ascidiidae Adams, 1858} \\
\hline Ascidia challengeri Herdman, 1882 & . & + & . & + & + & + & + & $1-4$ & 3,4 ? \\
\hline Ascidia meridionalis Herdman, 1880 & + & + & . & . & . & . & . & $1-4$ & \\
\hline Ascidia translucida Herdman, 1880 & . & + & . & . & . & . & + & $1-2$ & 3 \\
\hline Fam. Styelidae Sluiter, 1895 & & & & & & & & & . \\
\hline Alleocarpa bacca Ärnbäck, 1929 & + & . & . & . & . & . & . & 1 & . \\
\hline Alleocarpa bridgesi Michaelsen, 1900 & + & . & . & . & . & . & . & 1 & . \\
\hline Alleocarpa bigyna C. Monniot, 1978 & . & . & . & . & . & . & + & 1 & . \\
\hline Alleocarpa incrustans (Herdman, 1886) & + & + & . & . & . & . & . & 1 & . \\
\hline Cnemidocarpa barbata Vinogradova, 1962 & . & . & $\dot{.}$ & $\dot{.}$ & $\dot{0}$ & $\dot{+}$ & $\dot{+}$ & 1 & . \\
\hline Cnemidocarpa drygalskii (Hartmeyer, 1911) & . & . & . & . & + & + & + & $1-3$ & . \\
\hline Cnemidocarpa effracta C. Monniot, 1978 & . & . & . & . & . & . & + & 1 & . \\
\hline Cnemidocarpa eposi Monniot and Monniot, 1994 & $\dot{.}$ & $\dot{.}$ & $\dot{0}$ & $\dot{.}$ & $\dot{.}$ & $\dot{+}$ & . & 2 & . \\
\hline Cnemidocarpa minuta (Herdman, 1881) & . & . & . & . & . & . & + & 1 & . \\
\hline Cnemidocarpa nordenskiöldi (Michaelsen, 1898) & + & . & . & . & . & . & . & 1 & . \\
\hline Cnemidocarpa ohlini (Michaelsen, 1898) & + & . & . & . & . & . & . & $1-2$ & . \\
\hline Cnemidocarpa pfefferi (Michaelsen, 1898) & . & + & . & + & + & + & . & $1-\overline{3}$ & \\
\hline Cnemidocarpa robinsoni Hartmeyer, 1926 & + & . & : & . & . & . & . & 1 & $i$ \\
\hline Cnemidocarpa verrucosa (Lesson, 1830) & + & $\dot{+}$ & $\dot{+}$ & $\dot{+}$ & $\dot{+}$ & $\dot{+}$ & $\dot{+}$ & $1-2$ & . \\
\hline Cnemidocarpa victoriae Monniot and Monniot, 1983 & + & . & . & . & . & . & . & $1-2$ & . \\
\hline Dextrocarpa misanthropos C. Monniot, 1978 & . & . & . & . & . & . & + & 1 & . \\
\hline Dicarpa cornicula (C. Monniot, 1978) & $\dot{0}$ & $\dot{0}$ & $\dot{0}$ & $\dot{0}$ & $\dot{0}$ & $\dot{.}$ & + & 1 & . \\
\hline Dicarpa insinuosa (Sluiter, 1912) & . & + & . & . & + & + & . & $1-2$ & . \\
\hline Dicarpa tricostata (Millar, 1960) & . & + & . & . & . & + & . & 1 & . \\
\hline Oligocarpa megalorchis Hartmeyer, 1911 & . & . & . & . & . & . & + & 1 & . \\
\hline Polyzoa minor C. Monniot, 1970 & . & . & . & . & & . & + & 1 & . \\
\hline Polyzoa opuntia Lesson, 1830 & + & + & + & . & . & . & + & 1 & . \\
\hline Styela glans Herdman, 1881 & + & . & . & . & + & + & . & $1-4$ & . \\
\hline Styela malgalhaensis Michaelsen, 1898 & + & . & . & . & . & . & . & $1-3$ & . \\
\hline Styela mallei C. Monniot, 1978 & . & . & . & . & . & . & + & 1 & . \\
\hline Styela materna Monniot and Monniot, 1983 & . & + & + & . & . & . & . & $1-2$ & . \\
\hline Styela paessleri Michaelsen, 1898 & + & . & . & . & . & . & . & 1 & . \\
\hline Styela schmitti f. simplex Millar, 1960 & + & $\dot{.}$ & $\dot{0}$ & $\dot{.}$ & $\dot{.}$ & $\dot{.}$ & . & 1 & \\
\hline Styela squamosa Herdman, 1881 & ? & + & + & + & . & + & . & $1-4$ & $\dot{3}$ \\
\hline Styela talpina C. Monniot, 1978 & . & . & . & . & . & . & + & 1 & . \\
\hline Styela wandeli (Sluiter, 1911) & . & + & . & + & + & . & . & 1 & . \\
\hline
\end{tabular}


TABle 2 (CONT.). - Distribution of the Antarctic and Subantarctic ascidiofauna: (AC) Antarctic continent; (AP) Antarctic Peninsula; (MR) Magellan region; (SAI) Subantarctic islands (Gough, Bouvet, Prince Edward, Marion, Crozet, Kerguelen, Heard, McDonald, Macquarie); (SG) South Georgia Is.; (SO) South Orkney Is.; (SS) South Sandwich Is.. (Dr) depth range: (1) 0-200 m; (2) 201-500 m; (3) 501-1000 m); (4) $>1000 \mathrm{~m}$. (AZ) other zones: (1) Chile $\left(<42^{\circ} \mathrm{S}\right)$, Peru $\left(>17^{\circ} \mathrm{S}\right)$; (2) New Zealand sector $\left(<50^{\circ} \mathrm{S}\right)$; (3) Tasmania, Southern Australia; (4) South Africa, Namibia; (5) Cosmopolitan.

\begin{tabular}{|c|c|c|c|c|c|c|c|c|c|}
\hline & MR & SG & SS & SO & $\mathrm{AP}$ & $\mathrm{AC}$ & SAI & $\mathrm{Dr}$ & $\mathrm{AZ}$ \\
\hline \multicolumn{10}{|l|}{ Fam. Pyuridae Hartmeyer, 1904} \\
\hline Bathypera splendens Michaelsen, 1904 & + & . & . & + & + & + & . & $1-4$ & . \\
\hline Pyura bouvetensis (Michaelsen, 1904) & . & + & . & + & + & + & + & $1-3$ & \\
\hline Pyura chilensis Molina, 1782 & + & . & . & . & . & . & . & 1 & 1 \\
\hline Pyura discoveryi (Herdman, 1910) & . & + & . & + & + & + & . & $1-4$ & . \\
\hline Pyura georgiana (Michaelsen, 1898) & . & + & . & . & . & . & . & $1-2$ & . \\
\hline Pyura legumen (Lesson, 1830) & + & + & . & . & . & . & . & 1 & . \\
\hline Pyura lycoperdon Monniot and Monniot, 1983 & . & . & . & . & + & . & . & 1,2 & . \\
\hline Pyura obesa Sluiter, 1912 & . & . & . & + & + & . & . & $1-2$ & . \\
\hline Pyura paessleri (Michaelsen, 1900) & + & . & . & . & . & . & . & 1,2 & . \\
\hline Piura pilosa Monniot and Monniot, 1974 & . & . & . & . & . & . & + & 1 & . \\
\hline Pyura setosa (Sluiter, 1905) & . & . & . & + & + & + & . & $1-2$ & . \\
\hline Pyura squamata Hartmeyer, 1909 & . & + & . & + & + & + & + & $2-\overline{4}$ & . \\
\hline Pyura stubenrauchi (Michaelsen, 1900) & + & . & . & . & . & . & . & 1 & . \\
\hline Pyura tunica Kott, 1969 & . & . & . & . & . & + & . & 1 & . \\
\hline Fam. Molgulidae Lacaze-Duthiers, 1877 & & & & & & & & & . \\
\hline Eugyrioides guttula (Michaelsen, 1900) & + & . & . & . & . & . & . & 1 & . \\
\hline Eugyrioides kerguelensis (Herdman, 1881 & . & . & + & . & + & . & + & 1 & . \\
\hline Eugyrioides polyducta Monniot and Monniot, 1983 & . & + & + & . & + & + & . & 1 & . \\
\hline Eugyrioides septum (C. Monniot, 1978) & . & . & . & . & . & . & + & 1 & . \\
\hline Eugyrioides vannamei (C. Monniot, 1970) & + & . & . & . & . & . & . & 1 & . \\
\hline Gamaster vallatum C. Monniot, 1978 & . & . & . & . & . & . & + & 1 & . \\
\hline Molgula arnbackae C. Monniot, 1978 & . & + & + & . & . & . & + & 1 & . \\
\hline Molgula enodis (Sluiter, 1912) & . & . & . & + & + & + & . & 1 & . \\
\hline Molgula estadosi Monniot and Monniot, 1983 & + & . & . & . & . & . & . & 1 & . \\
\hline Molgula euplicata Herdman, 1923 & . & + & + & + & + & + & . & $1-3$ & . \\
\hline Molgula georgiana Michaelsen, 1900 & . & + & + & . & . & . & + & 1 & . \\
\hline Molgula hodgsoni Herdman, 1910 & . & + & . & + & + & + & . & $1-2$ & . \\
\hline Molgula macquariensis Kott, 1954 & . & . & . & . & . & . & + & 1 & . \\
\hline Molgula marioni Millar, 1960 & + & + & : & $:$ & : & . & + & 1 & \\
\hline Molgula mortenseni (Michaelsen, 1922) & + & + & . & . & . & . & . & 1 & $\dot{2}$ \\
\hline Molgula pedunculata Herdman, 1881 & . & + & + & + & + & + & + & $1-3$ & . \\
\hline Molgula pigafettae Monniot and Monniot, 1983 & + & . & . & . & . & . & . & $1-2$ & . \\
\hline Molgula pulchra Michaelsen, 1900 & + & + & + & . & . & . & + & $1-2$ & . \\
\hline Molgula pyriformis Herdman, 1881 & + & + & . & . & . & . & . & $1-4$ & . \\
\hline Molgula robini Monniot and Monniot, 1983 & . & + & : & $\dot{0}$ & $\dot{+}$ & + & $\dot{0}$ & $1-4$ & . \\
\hline Molgula setigera Ärnback-Christie-Linde, 1938 & + & . & . & . & . & . & . & 1 & . \\
\hline Molgula variazizi C. Monniot, 1978 & . & . & . & . & . & . & + & 1 & . \\
\hline Molguloides coronatum C. Monniot, 1978 & . & . & . & . & . & + & + & 2 & . \\
\hline Molguloides crinibus C. Monniot, 1978 & : & : & : & : & . & . & + & 2 & . \\
\hline Molguloides glans C. Monniot, 1978 & : & . & . & . & . & . & + & 2 & . \\
\hline Paraeugyrioides arnbackae (Millar, 1960) & . & . & + & . & + & + & . & $1-4$ & . \\
\hline Paraeugyrioides macquariensis Kott, 1954 & . & . & . & . & . & . & + & 1 & \\
\hline Paramolgula gregaria (Lesson, 1830) & + & + & . & . & . & . & . & $1-2$ & 1 \\
\hline Paramolgula canioi Monniot and Monniot, 1983 & + & . & . & . & . & . & . & 1 & . \\
\hline Total spp. & 70 & 55 & 28 & 34 & 63 & 58 & 73 & & \\
\hline
\end{tabular}

la eumyota, Ascidia challengeri, Cnemidocarpa drygalskii, Cnemidocarpa verrucosa, Pyura bouvetensis, P. squamata and Molgula pedunculata).

\section{Zoogeographical affinities}

The MDS analysis between zones (Fig. 2) for the 172 species listed in Table 2 shows the proximity between the Antarctic continent and the Antarctic Peninsula, and a clear separation from the Scotia Arc islands. The South Orkney Archipelago has a similar component to the Antarctic groups, and the ascidian fauna of the South Georgia and South Sandwich archipelagos is intermediate between that of the Antarctic, the Magellan region and the rest of the Subantarctic islands.

The comparison at species level of the main families (with percentages $>5 \%$ : Polyclinidae, Styelidae, Molgulidae, Pyuridae, Polycitoridae, Didemnidae, and Agneziidae; Table 3) confirms the differences between the archipelagos of the Scotia Arc. The South Orkney islands show similar percentages of Molgulidae (12\%), Styelidae (12\%), Polycitoridae (9\%) and Didemnidae (6\%) to the 


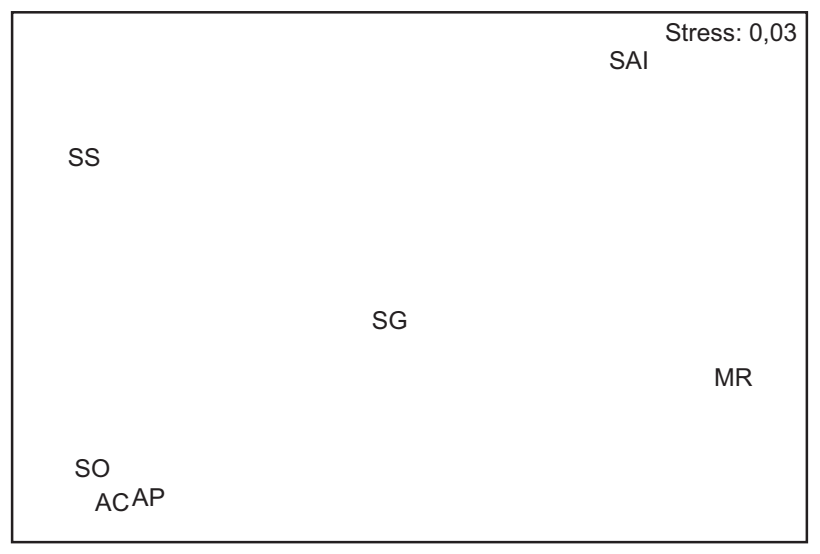

FIG. 2. - MDS analysis of the zones, based on the sampled ascidiofauna (distribution data completed with Monniot and Monniot, 1983). Legend: (AC) Antarctic Continent (without Antarctic Peninsula); (AP) Antarctic Peninsula (plus South Shetland Archipelago); (M) Magellan region; (SAI) Subantarctic Islands; (SG) South Georgia Archipelago; (SO) South Orkney Archipelago; (SS) South Sandwich Archipelago.

Antarctic region, and higher values than South Georgia and South Sandwich for Polyclinidae (32\%) and Pyuridae (18\%). The South Georgia and South Sandwich archipelagos have the lowest and the highest percentages of Polyclinidae (24-25\%) and Molgulidae (22-29\%) respectively among the Antarctic and Subantarctic zones; and Styelidae $(14-16 \%)$ is intermediate between the Antarctic (12-13\%) and Subantarctic (18-19\%) regions. However, Pyuridae and Didemnidae are relatively important in South Georgia (9\%), but scarce or never recorded (Pyuridae) in the South Sandwich Islands, whereas Agneziidae are well represented in the South Sandwich Islands (3 spp.), as in the Antarctic and Magellan regions (3-5 spp.). The lack or scarcity of the Pyuridae in the South Sandwich Islands is noteworthy, since this family presents big specimens and is easy to separate from the samples. Off the rest of the Subantarctic islands, Pyuridae are poorly represented (3 spp., with Pyura bouvetensis only reported from Bouvet island).

The biogeographical position of the ascidian fauna in the Scotia Arc is controversial. Reporting on this taxon, Kott $(1969,1969 a)$ included the Antarctic Peninsula (with the Bellingshausen Sea) and the Scotia Arc in the South Georgia Province (or western Antarctic subregion, cf. Knox, 1994). However, Millar (1971) and Monniot and Monniot (1983) consider the South Georgia Archipelago to occupy an intermediate position between the Antarctic and Magellan regions, and to be a separate province, whereas the South Orkney and South Sandwich islands belong to the Antarctic region, in accordance with Ekman (1967) and Briggs (1974).

The present study supports the inclusion of the Antarctic Peninsula and adjacent islands in the Antarctic Province and the division of the Scotia Arc into two sectors: the South Orkney Archipelago, more related to the Antarctic Province, and the South Georgia Archipelago, a separate area, intermediate between the Antarctic Province and the Magellan region. The position of the South Sandwich islands is doubtful. It is related on the one hand to the South Georgia Archipelago and the Subantarctic islands, and on the other hand to the Antarctic Province. Nevertheless, the Scotia Arc has been poorly sampled, and much more sampling effort (as the LAMPOS cruise) must be carried out to elucidate its biogeographical relationships.

\section{ACKNOWLEDGEMENTS}

The authors wish to express their gratitude to Ana Ramos and Ignacio Olaso, who were responsible for the 'Bentart' cruises, and our colleagues who

TABLE 3. - Distribution by number of species (percentage in brackets) of the littoral ascidian families: (AR) Antarctic region; (MR) Magellan region; (SG) South Georgia; (SS) South Sandwich; (SO) South Orkney; (SAI) Subantarctic islands; (SAR) Subantarctic region (data from Table 2).

\begin{tabular}{|c|c|c|c|c|c|c|c|}
\hline Families & MR & SAI & $\mathrm{SG}$ & SS & $\mathrm{SO}$ & AR & $\mathrm{AR}+\mathrm{SAR}$ \\
\hline Polyclinidae & $23(32.9)$ & $27(37.0)$ & 13 (23.6) & $7(25.0)$ & $11(32.4)$ & $29(37.2)$ & $57(33.1)$ \\
\hline Didemnidae & $5(7.1)$ & $5(6.8)$ & $5(9.1)$ & $1(3.6)$ & $2(5.9)$ & $4(5.1)$ & $11(6.4)$ \\
\hline Polycitoridae & $5(7.1)$ & $5(6.8)$ & $5(9.1)$ & $4(14.3)$ & $3(8.8)$ & $8(10.3)$ & $12(7.0)$ \\
\hline Cionidae & $2(2.9)$ & $1(1.4)$ & $1(1.8)$ & $1(3.6)$ & $1(2.9)$ & $2(2.6)$ & $3(1.7)$ \\
\hline Corellidae & $2(2.9)$ & $1(1.4)$ & $1(1.8)$ & - & $1(2.9)$ & $2(2.6)$ & $3(1.7)$ \\
\hline Agneziidae & $3(4.3)$ & $2(2.7)$ & $1(1.8)$ & $3(10.7)$ & $1(2.9)$ & $5(6.4)$ & $9(5.2)$ \\
\hline Ascidiidae & $1(1.4)$ & $2(2.7)$ & $3(5.5)$ & - & $1(2.9)$ & $1(1.3)$ & $3(1.7)$ \\
\hline Styelidae & 13 (18.6) & $13(17.8)$ & $9(16.4)$ & $4(14.3)$ & $4(11.8)$ & $10(12.8)$ & $31(18.0)$ \\
\hline Pyuridae & $5(7.1)$ & $3(4.1)$ & $5(9.1)$ & - & $6(17.6)$ & $8(10.3)$ & $14(8.2)$ \\
\hline Molgulidae & $11(15.7)$ & $14(19.2)$ & $12(21.8)$ & $8(28.6)$ & $4(11.8)$ & $9(11.5)$ & 29 (16.9) \\
\hline $\mathrm{N}^{\mathrm{o}}$ of species & 70 & 73 & 55 & 28 & 34 & 78 & 172 \\
\hline
\end{tabular}


participated in these expeditions. We also thank three anonymous referees who improved this paper. These cruises were carried out under the auspices of the Spanish Ministry of Education and Science (DGICYT ANT93-0996 and DGICYT ANT941161 projects).

\section{REFERENCES}

Arnaud, P., C.M. López, I. Olaso, F. Ramil, A.A. Ramos-Esplá and A. Ramos. - 1998. Semi-quantitative study of macrobenthic fauna in the region of the South Shetland Islands and the Antarctic Peninsula. Polar Biol., 19: 160-166.

Arntz, W.E. - 1999. Magellan-Antarctic: Ecosystems that drifted apart. Summary Review. Sci. Mar., 63(Suppl. 1): 503-511.

Arntz, W.E. and T. Brey. - 2003. The expedition Antarktis XIX/5 (LAMPOS) of RV "Polarstern" in 2002. Ber. Polarforsch. Meeresforsch., 462: 1-120.

Arntz, W.E., T. Brey and V.A. Gallardo. - 1994. Antarctic zoobenthos. Oceanogr. Mar. Biol. Ann. Rev., 32: 241-304.

Arntz, W.E., J. Gutt and M. Klages. - 1997. Antarctic marine biodiversity. In: B. Battaglia, J. Valencia and D.W.H. Walton (eds.), Antarctic communities: species, structure and survival, pp. 3-14. Cambridge University Press, Cambridge.

Briggs, J.C. - 1974. Marine zoogeography. McGraw-Hill, New York

Clarke, K.R. and R.M. Warwick. - 1994. Change in marine communities: an approach to statistical analysis and interpretation. Natural Environmental Research Council, UK.

Crame, J.A. - 1999. An evolutionary perspective on marine faunal connections between southernmost South America and Antarctica. Sci. Mar., 63(Suppl. 1): 1-14.

Dayton, P. - 1990. Polar benthos. In: W.O. Smith (ed.), Polar oceanography. Part B: Chemistry, biology, and geology, pp. 631-685 Academic Press, London.

Dell, R.K. - 1972. Antarctic benthos. Adv. Mar. Biol., 10: 1-216.

Ekman, S. - 1967. Zoogeography of the sea. Sidgwick and Jackson Watergate Eds., London.

Galéron, J., R.L. Herman, P.M. Arnaud, W.E. Arntz, S. Hain, and M. Klages. - 1992. Macrofaunal communities on the continental shelf and slope of the southeastern Weddell Sea, Antarctica. Polar Biol., 12: 283-290.

Gallardo, V.A. - 1987. The sublittoral macrofaunal benthos of the Antarctic shelf. Environ. Int., 13: 71-81.

Gili, J.M., R. Coma, C. Orejas, P.J. López-González and M. Zabala. - 2001. Are Antarctic suspension-feeding communities different from those elsewhere in the world? Polar Biol., 24: 473-485.

Hedgpeth, J.M. - 1969. Introduction to Antarctic zoogeography. In: V.C. Bushnell and J.W. Hedgpeth (eds.), Distribution of select- ed groups of marine invertebrates in waters south of $35^{\circ}$ latitude. Antarct. Map Folio Ser., Folio 11: 1-9. American Geological Society, New York.

Jazdzewski, K., W. Jurasz, W. Kittel, E Presler, P. Presler and J. Sicinski. - 1986. Abundance and biomass estimates of the benthic fauna in Admiralty Bay, King George Island, South Shetland Islands. Polar Biol., 6: 5-16.

Knox, G.A. - 1994. The biology of the Southern Ocean. Cambridge University Press, London.

Kott, P. - 1969. Antarctic Ascidiacea. Antarct. Res. Ser., 13: 1- 239.

Kott, P. 1969a. Ascidiacea. In: V.C. Bushnell and J.W. Hedgepeth (eds.). Distribution of selected groups of marine invertebrates in waters south of $35^{\circ}$ latitude. Antarct. Map Folio Ser., Folio 11: 43-44. American Geological Society, New York.

Kott, P. - 1971. Antarctic Ascidiacea II. Antarct. Res. Ser., 17: $11-82$.

Kowalke, J. - 1999. Filtration in Antarctic ascidians - striking a balance. J. Exp. Mar. Biol. Ecol., 242: 233-244.

Longhurst, A.R. - 1998. Ecological geography of the sea, 398 pp. Academic Press, Orlando, Florida.

Luján, I. and A.A. Ramos-Esplá. - 1996. Revisión de Synoicum adareanum Herdman, 1902 (Polyclinidade, Ascidiacea), ejemplares recolectados en la campaña antártica BENTART-94 (Isla Livingston, Islas Shetland del Sur). Res. VI Simp. Esp. Estud. Antárticos, Madrid: 52

Millar, R.H. - 1960. Ascidiacea. Discovery Rep., 30: 1-160

Millar, R.H. - 1971. The biology of ascidians. Adv. Mar. Biol., 9: $1-100$.

Monniot, C. and F. Monniot. - 1983. Ascidies antarctiques et subantartiques: morphologie et biogéographie. Mem. Mus. Natl. Hist. Nat., Paris, 215 (Sér. A): 1-135.

Monniot, C. and F. Monniot. - 1994. Ascidians collected in the Weddell Sea by the RV "Polarstern" (EPOS cruise leg 3). Bull. Mus. Natl. Hist. Nat., Paris, 4e sér., 16, section A, n ${ }^{\circ}$ 1: 13-37.

Ramos-Esplá, A.A. - 1991. Ascidias litorales del Mediterráneo ibérico. Faunística, ecología y biogeografía. Publicaciones de la Universidad de Alicante, Alicante, 405 pp. + VI pl.

Rauschert, M. - 1991. Ergebnisse der faunistischen Arbeiten im Benthal von King George Island (Südshetlandinseln, Antarktis). Ber. Polarfor., 76: 1-75.

Sahade, R., M. Tatián, J. Kowalke, S. Kühne and G.B. Esnal. - 1998. Benthic faunal associations on soft substrates at Potter Cove, King George Island, Antarctica. Polar Biol., 19(2): 85-91.

Sanamyan, K. and Schories, D. - 2003. Ascidians from the Strait of Magellan. Aqua, 7(3): 89-96.

Tatián, M., R. Sahade, M.E. Doucet and G.B. Esnal. - 1998. Ascidians (Tunicata, Ascidiacea) of Potter Cove, South Shetland Islands, Antarctica. Antarct. Sci., 10(2): 147-152.

Van Name, W.G. - 1945. The North and South American Ascidians. Bull. Am. Mus. Nat. Hist., 84: 1-476.

Varela, M. M. and A.A. Ramos-Esplá. - 2003. Contribution to the knowledge of the colonial ascidians (Chordata: Tunicata) of the Western Antarctic. 38 th Europ. Mar. Biol. Symp., Aveiro, 8-12 September 2003: 154. 
